\title{
SARS-CoV-2 seroprevalence in healthcare workers at a frontline hospital in Tokyo
}

\author{
Hiroshi FUKUDA \\ Juntendo University \\ Kuniaki SEYAMA \\ Juntendo University \\ Kanami ITO \\ Juntendo University \\ Satoshi HORI \\ Juntendo University \\ Mitsuru WAKITA \\ Juntendo University \\ Kaori SAITO \\ Juntendo University \\ Yuka SHIDA \\ Juntendo University \\ Rie NAGURA \\ Juntendo University \\ Mayu HASEGAWA \\ Juntendo University \\ Chiaki KANEMOTO \\ Juntendo University \\ Mayumi TOKUHARA \\ Juntendo University \\ Katsunobu OKAJIMA \\ Juntendo University \\ Yukio YOSHIKAWA \\ Juntendo University \\ Narimasa KATSUTA \\ Juntendo University \\ Takamasa YAMAMOTO \\ Juntendo University \\ Tomohiko Al \\ Juntendo University \\ Mayumi IDEI
}


Juntendo University

\section{Shigeki MISAWA}

Juntendo University

Toshio NAITO

Juntendo University

Takashi MIIDA

Juntendo University

Hiroyuki SATO

Juntendo University

Nobutaka HATTORI

Juntendo University

Yoko TABE ( $\nabla$ tabe@jjuntendo.ac.jp )

Juntendo University

Kazuhisa TAKAHASHI

Juntendo University

\section{Research Article}

Keywords: COVID-19, SARS-CoV-2, Healthcare workers, seroprevalence, Japan

Posted Date: October 23rd, 2020

DOI: https://doi.org/10.21203/rs.3.rs-96870/v1

License: (c) (i) This work is licensed under a Creative Commons Attribution 4.0 International License.

Read Full License

Version of Record: A version of this preprint was published at Scientific Reports on April 16th, 2021. See the published version at https://doi.org/10.1038/s41598-021-87688-9. 


\section{Abstract}

Healthcare workers (HCWs) are highly exposed to severe acute respiratory syndrome coronavirus 2 (SARS-CoV-2) infection. The aim of this study was to assess the seroprevalence of SARS-CoV-2 in HCWs working in a frontline hospital in Tokyo, Japan. In this observational cohort study, screening was offered to agreed HCWs, including medical, nursing, and others, as part of a mandatory health checkup. The screening test results and clinical characteristics of the participants were recorded. The antibody seroprevalence among the 4,147 participants screened from July 6 to August 21 2020, was $0.34 \%$ $(14 / 4,147)$. There was no significant difference in seroprevalence between frontline HCWs with a high exposure risk and HCWs in other settings with a low exposure risk. Of those seropositive for SARS-CoV-2, $64 \%$ (9/14) were not aware of any symptoms and had not previously been diagnosed with COVID-19. In conclusion, this study provides insights into the extent of infection and immune status in HCWs in Japan, which has a relatively low prevalence of COVID-19.

\section{Introduction}

COVID-19 caused by severe acute respiratory syndrome coronavirus 2 (SARS-CoV-2) has evolved into a pandemic with sustained human-to-human transmission. ${ }^{1}$ Clinical studies have found that approximately $5 \%$ of symptomatic cases develop severe symptoms and more than $80 \%$ of cases show mild symptoms, ${ }^{2}$ and that individuals with minimally symptomatic or asymptomatic infection carry the virus. ${ }^{3}$

Healthcare workers (HCWs) engage in clinical care of patients with suspected and confirmed COVID-19, are exposed to a high risk of infection with the disease. ${ }^{4}$ Therefore, HCWs working in regions severely affected by the COVID-19 pandemic have a high prevalence of SARS-CoV-2 infection detected by polymerase chain reaction ${ }^{5}$. Although clarifying the risk of COVID-19 among frontline HCWs is important for infection control, the actual situation, especially in regions that are less affected by COVID-19 has not yet been determined. Systematic screening for SARS-CoV-2 is an effective tool for surveillance of the pandemic, and the seroprevalence of SARS-CoV-2 among HCWs who are most exposed to SARS-CoV-2 infection is an effective indicator of the spread of SARS-CoV-2.

In this study, we aimed to determine the SARS-CoV-2 seroprevalence in HCWs working at a frontline hospital in the Tokyo area, in order to determine the prevalence of past infection, both symptomatic and asymptomatic, using a validated chemiluminescent assay ${ }^{6}$.

\section{Methods}

\section{Study design and participants}

This observational cohort study was conducted from July 6 to August 21 as part of a mandatory health checkup of employees working at the Juntendo University Hospital and employees and students of the Juntendo University Graduate School of Medicine, Tokyo, Japan. A total of 4,147 participants underwent 
antibody identification from blood specimens. All participants completed a web-based questionnaire on their medical history and health status. Detailed medical history interviews regarding possible COVID-19 infections were conducted by clinical interviewers to the SARS-CoV-2 antibody-positive individuals. The participants were classified as follows: medical doctors $(n=1,111)$, nurses $(n=1,308)$, laboratory technicians $(n=236)$, paramedics $(n=314)$, administrative staff $(n=510)$, researchers $(n=632)$, and others $(n=36)$. To better classify the individual risk rate, three categories were identified. High exposure-risk (HR) occupation: frontline HCWs, including medical doctors and nurses. Medium exposure-risk (MR) occupation: non-frontline paramedics or laboratory personnel transporting or handling specimens from patients. Low exposure-risk (LR) occupation: administrative staff of the hospital, researchers, and others who may have minimal chance of exposure. All healthcare personnel who had any contact with infected/suspected cases of COVID-19, wore proper personal protective equipment recommended by US Centers for Disease Control and Prevention (CDC) (https://www.cdc.gov/coronavirus/2019ncov/hcp/using-ppe.html).

This research complied with all relevant national regulations and institutional policies, was conducted in accordance with the tenets of the Helsinki Declaration, and was approved by the Institutional Review Board (IRB) at Juntendo University (IRB \#20-089). Informed consent was obtained from all the study participants.

\section{Measurement of SARS-CoV-2}

We measured SARS-CoV-2 antibodies with a fully automated Cobas e801 analyzer (Roche Diagnostics, Basel, Switzerland) using the Elecsys Anti-SARS-CoV-2 electrochemiluminescence immunoassay (Roche Diagnostics). The electrochemiluminescence immunoassay uses a modified double-antigen sandwich immunoassay with recombinant nucleocapsid protein $(\mathrm{N})$, which enables quantitative detection of late, mature, high-affinity immunoglobulin $\mathrm{A}$, immunoglobulin $\mathrm{M}$, and immunoglobulin G SARS-CoV-2 antibodies. Results are reported as numeric values in the form of a cutoff index (COl; signal sample/cutoff) with qualitative results reactive ( $\mathrm{COI} \geq 1.0$; positive). The analytical and clinical performance of the assay have been evaluated and are described elsewhere. ${ }^{6}$

PCR-based testing of SARS-CoV-2 for the diagnosis of COVID-19 was performed using the LightMix Modular SARS-CoV (COVID19) N-gene and E-gene assay (Roche Diagnostics, Tokyo, Japan) or the 2019 Novel Coronavirus Detection Kit (Shimadzu, Kyoto, Japan).

\section{Statistical analysis}

Statistical analyses were performed using Stat Flex for Windows (ver. 6.0; Artech, Osaka, Japan). Chisquare tests or Fisher's exact tests were used to evaluate the significance of differences in proportions between groups. The Mann-Whitney $U$ test was used to compare the COI between the symptomatic and asymptomatic HCWs with SARS-CoV-2 antibodies. A 2-tailed $p$ value of $<0.05$ was considered statistically significant. 


\section{Results}

A total of 4,147 HCWs at the Juntendo University Hospital and employees of the Juntendo University Graduate School of Medicine participated in this study. Table 1 shows the age and sex composition of the study participants. The mean age was 36.8 years (standard deviation 12.0) years. Of the participants, $36.1 \%$ were male and $63.9 \%$ were female.

Of the 4147 participants, 14 showed positive results for SARS-CoV-2 antibody, yielding a seroprevalence of $0.34 \%$ (95\% confidence interval [Cl]: $0.18-0.57 \%$ ) (Table 2). There was no significant difference in seroprevalence between male $(0.33 \%, 5 / 1,498)$ and female participants $(0.43 \%, 13 / 2,649)(p=0.556)$, and no significant association with age; $20-29$ years $(0.27 \%, 4 / 1461), 30-39$ years $(0.40 \rrbracket, 5 / 1245), 40-49$ years $(0.39 \%, 3 / 776), 50-59$ years $(0.24 \%, 1 / 424), 60-69$ years $(0.52 \%, 1 / 193)$, over 70 years $(0 \%, 0 / 48)$ $(p=0.891)$.

The seroprevalence of SARS-CoV-2 in HCWs in different job categories is shown in Table 2. The seroprevalence was highest in paramedics and lowest in laboratory workers. There was no significant difference in seroprevalence among frontline HCWs; HR $0.33 \%$, MR $0.36 \%$, and LR $0.34 \%$. In addition, none of the HCWs working in dedicated the COVID-19 outpatient clinic and wards, including medical doctors and nurses, were positive for SARS-CoV-2 antibody.

Transmission can occur from patient to HCW and between HCWs. We therefore interviewed all the seropositive participants about these interactions, and confirmed that one of the paramedics was infected at work. Among the 14 seropositive participants, only five (36\%) had been diagnosed with SARSCoV-2 infection by PCR and had experienced some COVID-19 symptoms (Table 3). Of note, two participants who had been diagnosed with SARS-CoV-2 infection by PCR were negative for SARS-CoV-2 antibodies. The numeric values of the cutoff index (COI) for the SARS-CoV-2 antibody were divided into a group showing high values $(\geq 10)$ and a group showing low values near the cutoff $(1-10)$. Of the five participants who had been diagnosed with symptomatic COVID-19, confirmed by PCR, only $1(20 \%)$ showed a low $\mathrm{COI}$, and among nine asymptomatic participants, $67 \%(6 / 9)$ were antibody-positive with a low $\mathrm{COI}$ (Figure 1). The median $\mathrm{COI}$ of the symptomatic and asymptomatic groups was 28.2 (interquartile range, IQR 13.5 - 76.7) and 2.5 (IQR 1.9 - 102.8), respectively. However, this difference in antibody levels between symptomatic and asymptomatic groups was not statistically significant because of the small number of positive participants, and the large variation in antibody titers.

\section{Discussion}

This study demonstrated a low seroprevalence of SARS-CoV-2 antibodies in HCWs working at a frontline hospital in the Tokyo area after the first wave of the COVID-19 epidemic in Japan from March to May, 2020 .

The lower prevalence of SARS-CoV-2 antibodies observed in HCWs reflects the lower circulation of SARSCoV-2 in Japan compared to some other countries where more canses were reported. A high incidence of 
infection has been reported among COVID-19 frontline medical staff in China ${ }^{7}$ and a higher prevalence of SARS-CoV-2 antibodies has been reported in HCWs in England, Belgium, and the United States $\left(6 \%{ }^{8}\right.$, $6.4 \%^{9}$, and $33 \%{ }^{10}$, respectively).

The Japanese government survey on the SARS-CoV-2 antibody-positive rate from June 1 st to 7 th, targeting local residents in Tokyo $(n=1,971)$, and other representative cities in Osaka $(n=2,970)$, and Miyagi $(n=3,009)$ prefectures using the Elecsys Anti-SARS-CoV-2 immunoassay, demonstrated an antibody prevalence of $0.30 \%, 0.34 \%$, and $0.23 \%$, respectively (https://www.mhlw.go.jp/stf/newpage_11892.html). Our survey with the same assay system revealed no significant difference in the prevalence of seropositivity between frontline HCWs and the general public in the Tokyo area.

We further observed no significant difference in the seroprevalence of SARS-CoV-2 antibodies between frontline HCWs and those working in other settings, which is inconsistent with previous reports that showed a higher seroprevalence in HCWs with higher exposure to COVID-19 ${ }^{11}$. These results may be partly due to the limited size of the epidemic in Japan, with ample access to protective equipment and sufficient time to prepare to prevent transmission. During the COVID-19 pandemic, the provision of adequate health care to patients is highly reliant on HCWs who are safe and well protected ${ }^{12}$. Along with continuing to observe the recommend standard protective precautions per CDC guidelines, knowing the seroprevalence of SARS-CoV-2 antibodies in HCWs helps to estimate the safety of their work environment.

Of the seropositive HCWs, $64 \%$ reported an absence of any signs or symptoms in the previous three months, which is consistent with previous studies that showed that almost $90 \%$ of infections among HCWs in the United States were asymptomatic ${ }^{13,14}$. Our results show that some individuals with SARs_CoV-2 infection develop antibodies without displaying signs of disease. The nature and strength of innate and adaptive immune responses in such cases require further investigation. A better understanding would be obtained by ongoing surveillance.

Systematic surveillance of the proportion of seropositive HCWs is an important indicator of the spread of SARS-CoV-2 and is useful for assessing the effectiveness of infection prevention in the medical setting. To our knowledge, this study is one of the largest systematic screening study of SARS-CoV-2 seroprevalence in HCWs in Japan.

The strengths of this study include: (1) the study scope and screening for SARS-CoV-2 antibodies detects all cases of SARS-CoV-2 infection to date; (2) the study had high participation of HCWs who submitted the mandatory web-based questionnaire; and (3) participants were not selected for screening on the basis of the presence of symptoms. Limitations include: (1) it being a single survey in a single institution that cannot be considered representative of the entire healthcare workforce in the Tokyo area, (2) the relationship between currently measured antibodies and neutralizing activity against SARS-CoV-2 were not evaluated. Therefore, we plan to perform a follow-up survey for five more years to better understand 
the duration of immune response and protection from reinfection by measuring neutralizing antibodies by confirmation assay (e. g., plaque reduction neutralization test).

In conclusion, this survey provides an insight into the extent of infection and immune status in the Japanese HCW population (i.e., a Juntendo University Hospital, Tokyo area); (1) an overall low seroprevalence of SARS-CoV-2 antibodies, (2) the frontline HCWs working in hospitals and HCWs working on a dedicated COVID-19 ward showed no significant difference in seroprevalence compared with other HCWs with low exposure risk, (3) more than half of seropositive HCWs had no symptoms attributable to SARS-CoV-2, which strengthens the hypothesis that "asymptomatic" cases have developed a specific immune response againstSARS-CoV-2.

Our results have relevance for public health policy to control virus spread in a region with a low intensity COVID-19 epidemic.

\section{Declarations}

\section{Acknowledgement}

The authors would like to thank Jean Ikawa, Masanobu Hinata, Masayoshi Chonan, Koji Tsuchiya, Takaaki Kawakami, Toshihiro Takahashi, Yoshie Hosaka, Maiko Yuri for technical support. for technical support and data collection.

\section{Author contributions}

$\mathrm{HF}, \mathrm{KS}, \mathrm{YT}$ designed the study. KS, MW, TY carried out data collection and analysis. YT, TA, MI performed data analysis and wrote the manuscript. $K I, Y S, R N, M H, C K, M T, K O, Y Y, N K$ contributed to sample collection. $\mathrm{SH}, \mathrm{TN}, \mathrm{YS}, \mathrm{NH}, \mathrm{KT}$ discussed experiments and contributed to data interpretation; and all authors critically reviewed and revised the manuscript for content and approved the manuscript for publication.

\section{Competing interests}

Roche Diagnostics provided reagents for Elecsys ${ }^{\circledR}$ Anti-SARS-CoV-2 measurements free of charge. The company did not play a role in (1) the design of the study; (2) data collection, analysis and interpretation.

\section{Informed consent and Ethical approval.}

Ethical approval: Research involving human subjects complied with all relevant national regulations, institutional policies and is in accordance with the tenets of the Helsinki Declaration (as revised in 2013), and informed consent was obtained in accordance with the Juntendo University Hospital IRB-approved protocol (IRB \#20-089).

\section{References}


1 Zhu, N. et al. A Novel Coronavirus from Patients with Pneumonia in China, 2019. N Engl J Med 382, 727-733, doi:10.1056/NEJMoa2001017 (2020).

2 Yang, X. et al. Clinical course and outcomes of critically ill patients with SARS-CoV-2 pneumonia in Wuhan, China: a single-centered, retrospective, observational study. Lancet Respir Med 8, 475-481, doi:10.1016/s2213-2600(20)30079-5 (2020).

3 Day, M. Covid-19: four fifths of cases are asymptomatic, China figures indicate. Bmj 369, m1375, doi:10.1136/bmj.m1375 (2020).

4 Ng, K. et al. COVID-19 and the Risk to Health Care Workers: A Case Report. Ann Intern Med 172, 766-767, doi:10.7326/l20-0175 (2020).

5 Remuzzi, A. \& Remuzzi, G. COVID-19 and Italy: what next? The Lancet 395, 1225-1228, doi:10.1016/S0140-6736(20)30627-9 (2020).

6 Egger, M. et al. Comparison of the Elecsys ${ }^{\circledR}$ Anti-SARS-CoV-2 immunoassay with the EDI ${ }^{\mathrm{m}}$ enzyme linked immunosorbent assays for the detection of SARS-CoV-2 antibodies in human plasma. Clin Chim Acta 509, 18-21, doi:10.1016/j.cca.2020.05.049 (2020).

7 Zhan, M., Qin, Y., Xue, X. \& Zhu, S. Death from Covid-19 of 23 Health Care Workers in China. N Engl J Med 382, 2267-2268, doi:10.1056/NEJMc2005696 (2020).

8 Poulikakos, D., Sinha, S. \& Kalra, P. A. SARS-CoV-2 antibody screening in healthcare workers in a tertiary centre in North West England. J Clin Virol 129, 104545, doi:10.1016/j.jcv.2020.104545 (2020).

9 Steensels, D. et al. Hospital-Wide SARS-CoV-2 Antibody Screening in 3056 Staff in a Tertiary Center in Belgium. Jama 324, 195-197, doi:10.1001/jama.2020.11160 (2020).

10 Moscola, J. et al. Prevalence of SARS-CoV-2 Antibodies in Health Care Personnel in the New York City Area. Jama 324, 893-895, doi:10.1001/jama.2020.14765 (2020).

11 Mansour, M. et al. Prevalence of SARS-CoV-2 Antibodies Among Healthcare Workers at a Tertiary Academic Hospital in New York City. J Gen Intern Med 35, 2485-2486, doi:10.1007/s11606-020-05926-8 (2020).

12 Zhan, M., Anders, R. L., Lin, B., Zhang, M. \& Chen, X. Lesson learned from China regarding use of personal protective equipment. Am J Infect Control, doi:10.1016/j.ajic.2020.08.007 (2020).

13 Sutton, D., Fuchs, K., D'Alton, M. \& Goffman, D. Universal Screening for SARS-CoV-2 in Women Admitted for Delivery. N Engl J Med 382, 2163-2164, doi:10.1056/NEJMc2009316 (2020).

14 Baggett, T. P., Keyes, H., Sporn, N. \& Gaeta, J. M. Prevalence of SARS-CoV-2 Infection in Residents of a Large Homeless Shelter in Boston. Jama 323, 2191-2192, doi:10.1001/jama.2020.6887 (2020). 


\section{Tables}

Table 1: Baseline characteristics of the study participants

$$
\text { participants }(\mathrm{n}=4,147)
$$

\begin{tabular}{ll}
\hline Age & \\
$20-29$ & $1,461(35.2 \%)$ \\
$30-39$ & $1,245(30.0 \%)$ \\
$40-49$ & $776(18.7 \%)$ \\
$50-59$ & $424(10.2 \%)$ \\
$60-69$ & $193(4.7 \%)$ \\
$70-$ & $48(1.2 \%)$
\end{tabular}

Sex

$\begin{array}{ll}\text { Male } & 1,498(36.1 \%) \\ \text { Female } & 2,649(63.9 \%)\end{array}$

Data are $\mathrm{n}$ or $\mathrm{n}(\%)$

Table 2: Antibody seroprevalence among healthcare workers according to the risk of exposure and professional category

\begin{tabular}{|c|c|c|c|c|}
\hline \multirow{2}{*}{$\begin{array}{l}\text { participants } \\
\text { High exposure risk (HR) }\end{array}$} & \multicolumn{3}{|l|}{ participants } & \multirow{4}{*}{$\begin{array}{l}\text { antibody positive } \\
5(0.45 \%)\end{array}$} \\
\hline & & & & \\
\hline & Medical Doctors & $(\mathrm{n}=1,111)$ & & \\
\hline & Nurses & $(\mathrm{n}=1,308)$ & $3(0.23 \%)$ & \\
\hline \multicolumn{5}{|c|}{ Medium exposure risk (MR) } \\
\hline & Laboratory personnel & $(n=236)$ & $0 \quad(0.00 \%)$ & \\
\hline & Paramedics & $(\mathrm{n}=314)$ & $2(0.64 \%)$ & \\
\hline \multicolumn{5}{|c|}{ Low exposure risk (LR) } \\
\hline & Administrative staff & $(n=510)$ & $3(0.59 \%)$ & \\
\hline & Researchers & $(n=632)$ & & $1(0.16 \%)$ \\
\hline & Other & $(n=36)$ & & $0 \quad(0.00 \%)$ \\
\hline All & & $(\mathrm{n}=4,147)$ & & $14(0.34 \%)$ \\
\hline
\end{tabular}


Table 3: Line listing of the clinical and demographic characteristics and the antibody levels of the healthcare workers who tested positive for SARS-CoV-2 antibodies

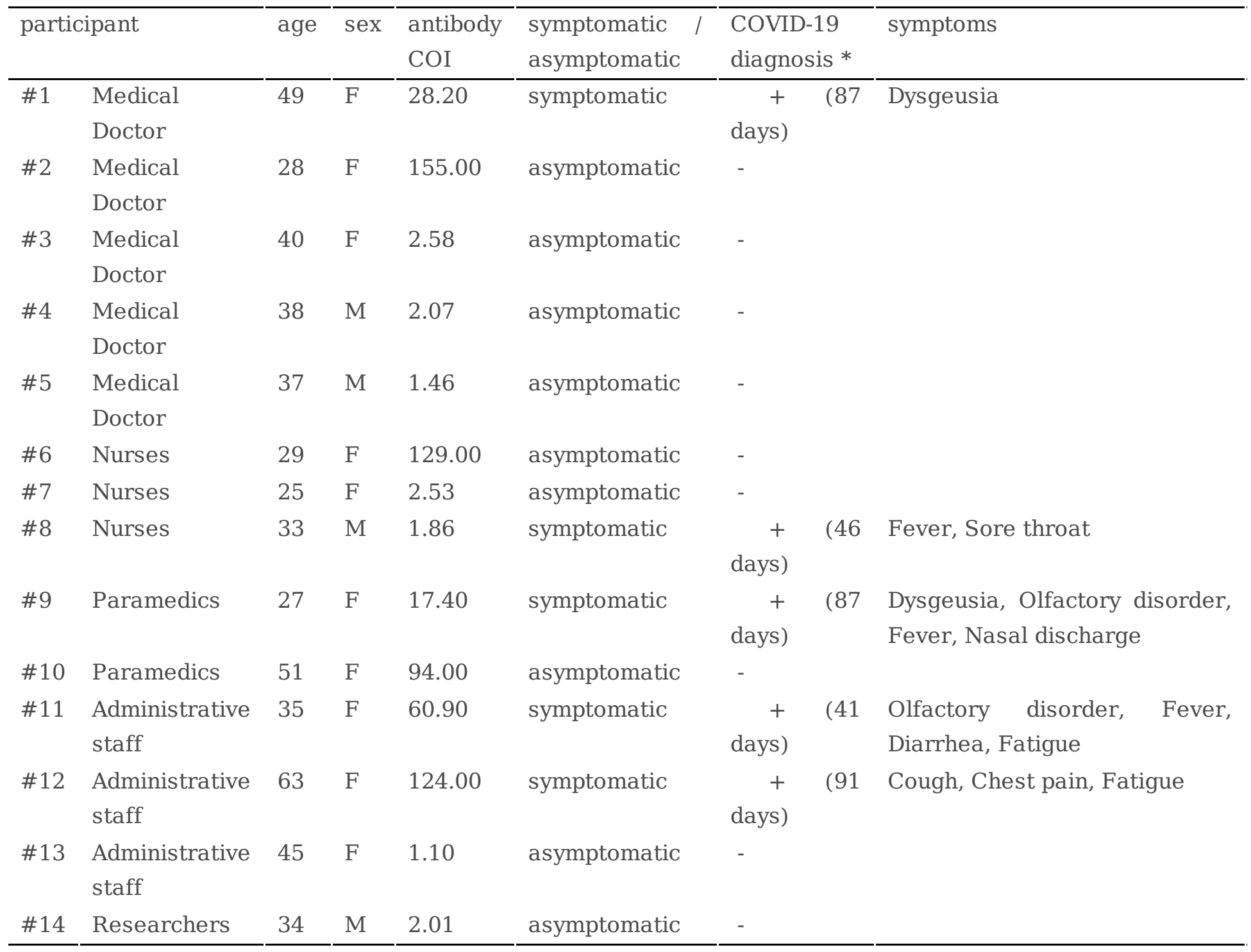

*COVID-19 diagnosis has been done by RT-PCR (days from diagnosis date to antibody detection date)

\section{Figures}




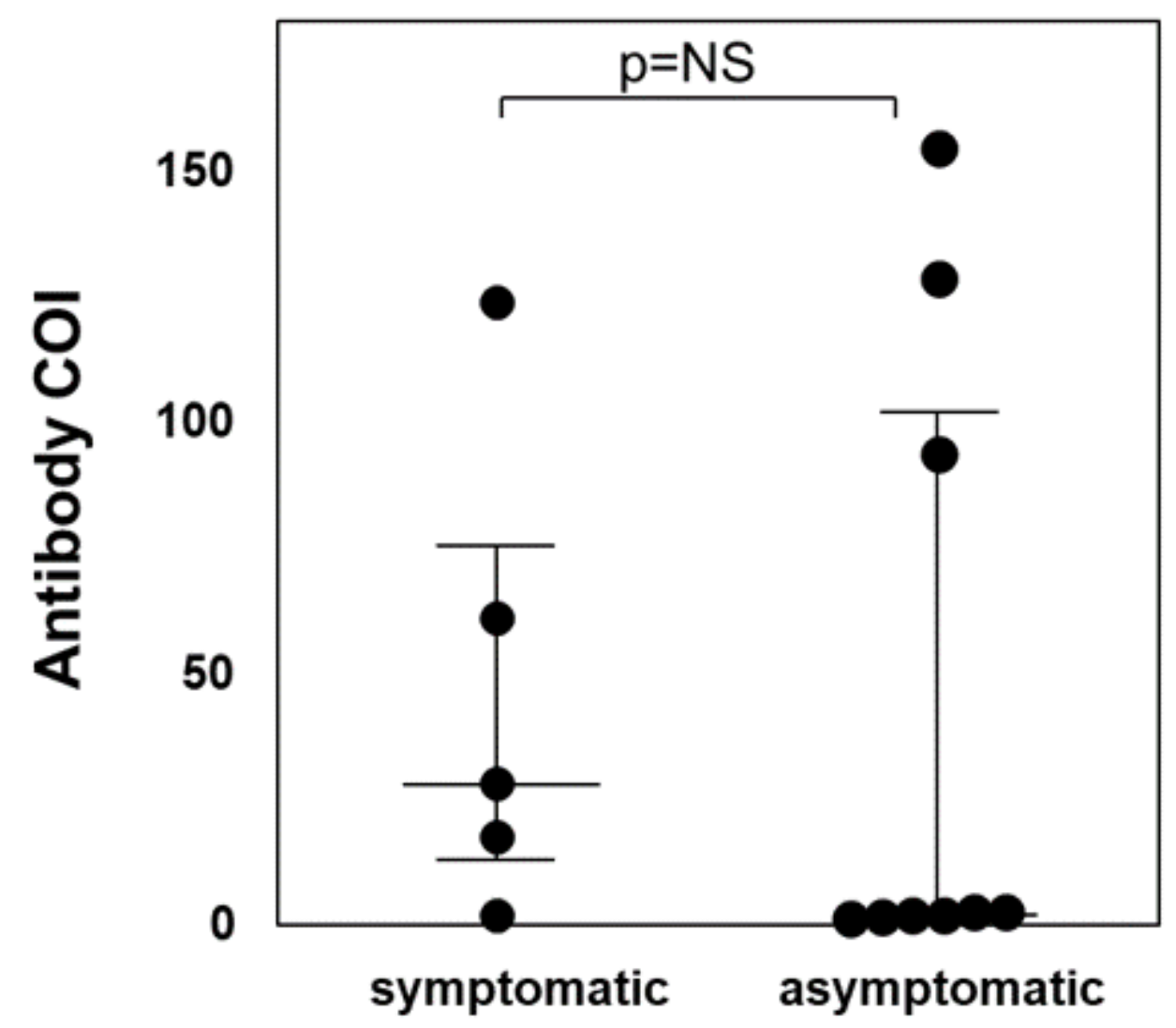

Figure 1

Comparison of SARS-CoV-2 antibody levels among symptomatic and asymptomatic healthcare workers with SARS-CoV-2 antibodies. Antibody levels were quantified using the anti-SARS-CoV-2 antibody index (COI) in participants who were positive by the Elecsys Anti-SARS-CoV-2 electrochemiluminescence immunoassay. A COI $\geq 1.0$ was considered positive. The median values of the $\mathrm{COI}$ with lower quartile and upper quartile are shown according to symptom status. The difference in the median antibody level between the symptomatic and asymptomatic groups was not significant. 